\title{
Identification of antiplasmodial triterpenes from Keetia species using NMR-based metabolic profiling
}

\author{
Rafael Teixeira Freire ${ }^{1} \cdot J^{\prime}$ oanne Bero ${ }^{2} \cdot$ Claire Beaufay $^{2} \cdot$ Denise Medeiros Selegato $^{1} \cdot$ Aline Coqueiro $^{1}$. \\ Young Hae Choi ${ }^{1,3}$. Joëlle Quetin-Leclercq ${ }^{2}$
}

Received: 21 November 2018 / Accepted: 4 February 2019 / Published online: 21 February 2019

(c) The Author(s) 2019

\begin{abstract}
Introduction The increase in multidrug resistance and lack of efficacy in malaria therapy has propelled the urgent discovery of new antiplasmodial drugs, reviving the screening of secondary metabolites from traditional medicine. In plant metabolomics, NMR-based strategies are considered a golden method providing both a holistic view of the chemical profiles and a correlation between the metabolome and bioactivity, becoming a corner stone of drug development from natural products. Objective Create a multivariate model to identify antiplasmodial metabolites from ${ }^{1} \mathrm{H}$ NMR data of two African medicinal plants, Keetia leucantha and $K$. venosa.

Methods The extracts of twigs and leaves of Keetia species were measured by ${ }^{1} \mathrm{H}$ NMR and the spectra were submitted to orthogonal partial least squares (OPLS) for antiplasmodial correlation.

Results Unsupervised ${ }^{1} \mathrm{H}$ NMR analysis showed that the effect of tissues was higher than species and that triterpenoids signals were more associated to Keetia twigs than leaves. OPLS-DA based on Keetia species correlated triterpene signals to K. leucantha, exhibiting a higher concentration of triterpenoids and phenylpropanoid-conjugated triterpenes than $K$. venosa. In vitro antiplasmodial correlation by OPLS, validated for all Keetia samples, revealed that phenylpropanoid-conjugated triterpenes were highly correlated to the bioactivity, while the acyclic squalene was found as the major metabolite in low bioactivity samples.

Conclusion NMR-based metabolomics combined with supervised multivariate data analysis is a powerful strategy for the identification of bioactive metabolites in plant extracts. Moreover, combination of statistical total correlation spectroscopy with 2D NMR allowed a detailed analysis of different triterpenes, overcoming the challenge posed by their structure similarity and coalescence in the aliphatic region.
\end{abstract}

Keywords Keetia leucantha $\cdot$ Keetia venosa $\cdot$ In vitro antiplasmodial activity $\cdot$ NMR-based metabolomics $\cdot$ Triterpenes . STOCSY

Rafael Teixeira Freire and Joanne Bero have contributed equally to this work.

Electronic supplementary material The online version of this article (https://doi.org/10.1007/s11306-019-1487-4) contains supplementary material, which is available to authorized users.

Young Hae Choi

y.choi@chem.leidenuniv.nl

$\bowtie$ Joëlle Quetin-Leclercq

joelle.leclercq@uclouvain.be

Natural Products Laboratory, Institute of Biology, Leiden University, Sylviusweg 72, 2333 BE Leiden, The Netherlands

2 Pharmacognosy Research Group, Louvain Drug Research Institute, Université catholique de Louvain, UCLouvain,
Avenue E. Mounier, 72, B1.72.03, B- 1200 Brussels, Belgium

3 College of Pharmacy, Kyung Hee University, Seoul 02447, Republic of Korea 


\section{Introduction}

Every year several 100 million of people get malaria, 1.2 million of which die. Even if a reduction of $37 \%$ cases has been recently reported, it remains the most severe parasitic disease worldwide, particularly for children under the age of five and pregnant women (World Health Organisation (WHO) 2018). Despite efforts to develop vaccines, the antigenic variability of these parasites allows only a partial and decreasing protection against clinical malaria and only in children and infants (Olotu et al. 2016).

In addition, malaria therapy faces some emerging problems such as parasite multidrug resistance, mosquito resistance to insecticides and the shortage of the time and money required for the development of new synthetic and natural leads (Achan et al. 2018; Woodrow and White 2017). The need to discover new prototypes of drugs is thus very important. In this context, natural products provide a high degree of lead-/drug-similarity, remaining undoubtedly the best source of native drugs or structural templates for antimalarial compounds development (Cargnin et al. 2018; Da Silva et al. 2013). A recent review reporting the new drugs available on the market during the last 34 years showed that approximately $60 \%$ of these new antiparasitic drugs have a natural origin/pharmacophore (Newman and Cragg 2016).

To date, the natural product database NAPRALERT has reported over 152 plant genera with a historical record of antimalarial properties, thus offering unlimited possibilities for the identification of novel hits/targets (Graham and Farnsworth 2010; Mojab 2012; Wells 2011). So far, however, the chemical and biological potential of these medicinal plants remains little explored.

When attempting to screen plant-matrixes, the large dynamic range and diversity of metabolites still hamper their identification and biological correlation. In the past, bio-guided fractionation was often used to identify active compounds. However, these reductionist procedures often lead to a laborious, time-consuming, non-comprehensive and expensive process of isolation and purification (Chen et al. 2015; Pezzuto 1997). Recently, metabolomics emerged as a fast alternative screening method to correlate chemical and biological data of natural products (Yuliana et al. 2011). Moreover, in an untargeted analysis of medicinal plants, metabolomics allows a broad range of chemical comparison between samples (Queiroz et al. 2009), prioritizing the identification of active molecules and, consequently, improving the process of chemotype and sample selection (Abreu et al. 2017; Kumar et al. 2014; Stermitz et al. 2000).

Considering their coverage of metabolites, sensitivity and resolution, NMR and MS methodologies are currently the most popular methods and are complementarily used for detailed chemical information on many biological systems.
Mass spectrometry offers high sensitivity and resolution with some structure information deduced from accurate mass, fragmentation patterns or isotope distribution (Lei et al. 2011; Lindon et al. 2007; Markley et al. 2017). On the other hand, NMR provides a highly reproducible and nondestructive analysis with minimal sample preparation, enabling the detailed elucidation of a wide range of metabolic groups, including isomers and compounds that are difficult to ionize or derivatize for MS (Markley et al. 2017).

NMR-based chemical profiling has often been used in plant-metabolomics for chemotaxonomy (Kim et al. 2010a), quality control (Yang et al. 2006) and as a bioactivity screening method (Cardoso-Taketa et al. 2008; Yuliana et al. 2011) following a suggested protocol (Kim et al. 2010a). In addition to simple one-dimensional ${ }^{1} \mathrm{H}$ NMR analysis, various two-dimensional experiments can be applied to ensure a broad interpretation of the data, providing detailed structure elucidation and quantification (Brennan 2014; Markley et al. 2017; Nagana Gowda and Raftery 2015). However, the difficulty of hyphenating NMR to chromatographic separation systems and its low-sensitivity has led to some inherent problems in chemical profiling research, including spectral congestion and the identification of minor metabolites (Kim et al. 2010b). For example, the elucidation of selected ${ }^{1} \mathrm{H}$ resonances in the congested region, requires a previous signal deconvolution (Emwas 2015). Two-dimensional NMR spectra, e.g. J-resolved (Huang et al. 2015; Ludwig and Viant 2010) and ${ }^{1} \mathrm{H}-{ }^{13} \mathrm{C}-\mathrm{HSQC}$ (Öman et al. 2014; Xi et al. 2008) have been applied to the analysis of complex spectra, in which an additional axis provides a higher resolution that can reveal invisible signals or peak purity. Furthermore, recent advances for overcoming these highly coalescence signals can also be based on the use of post-analytical deconvolution algorithms, assisting on detection and quantification of metabolites without the use of separation techniques. One example is statistical total correlation spectroscopy (STOCSY), which enables digital separation not limited to the usual connectivity of two-dimensional NMR methods and takes advantage of the multicollinearity of the signals intensity in a ${ }^{1} \mathrm{H}$ NMR spectra (Cloarec et al. 2005).

In this study, NMR-based metabolomics with STOCSY was applied to a model of an antiplasmodial medicinal plant in order to identify bioactive metabolites involved in the activity. Recently, dichloromethane extracts of Keetia leucantha, an African antimalarial plant listed by the Direction de la Protection Sanitaire of the Benin Ministry of Health, showed promising in vitro antiplasmodial activity against both susceptible and resistant Plasmodium strains and in vivo antimalarial efficacy without any acute toxicity at therapeutic doses. This activity has been related to active triterpenes (Beaufay et al. 2017, 2019; Bero et al. 2009, 2013). In this paper, we report the use of NMR-based metabolomics and the implementation of a post-analytical 
deconvolution algorithm to identify antiplasmodial metabolites from ${ }^{1} \mathrm{H}$ NMR data of $K$. leucantha and another related species, $K$. venosa. Specifically, dichloromethane extracts of twigs and leaves of both Keetia species were measured by ${ }^{1} \mathrm{H}$ NMR and the spectra were submitted to orthogonal partial least square modeling (OPLS). Correlation with bioactivity was performed according to their in vitro antiplasmodial response (expressed by $\mathrm{IC}_{50}$ ) against chloroquine sensitive and -resistant Plasmodium falciparum strains (3D7 and W2, respectively). For metabolite elucidation, targeted OPLS signals were submitted to STOCSY and confirmed by 2D-NMR experiments.

\section{Materials and methods}

\subsection{Plant material}

Keetia venosa (Oliv.) Bridson (17 twigs and 17 leaves) and K. leucantha (K. Krause) Bridson (27 twigs and 27 leaves) (Rubiaceae) were collected by Dr Agbani, a specialized botanist from University of Abomey-Calavi in Benin over the period of 2014-2015 in the Republic of Benin, West Africa, in different geographical locations in Donga or Ouémé/Zou departments, respectively: Djougou, Bassila and Belefunga for $K$. venosa and Adjara, Tako (Porto Novo), Lama and Katagon for K. leucantha.

The leaves and twigs samples were air-dried immediately after collection. Herbarium samples, prepared locally during the collection, and further identified by Dr. Olivier Lachenaud, compared with voucher specimens, have been deposited at the Herbarium of the National Botanic Garden of Belgium (Vouchers BR0000005087129 and BR00000014420382 for K. leucantha and BR0000005087228, BR0000005087242, BR00000014420443 and BR00000014420375 for $K$. venosa).

\subsection{Sample preparation}

Dried and ground plant material (200 mg) was vortexed and ultrasonicated for $30 \mathrm{~s}$ and $15 \mathrm{~min}$, respectively, with $2.0 \mathrm{~mL}$ of dichloromethane. For NMR- and biological analysis, the filtered (with cotton wool) and dried dichloromethane extracts were dissolved in $750 \mu \mathrm{L}$ of $\mathrm{CD}_{3} \mathrm{OD}$, vortexed for $30 \mathrm{~s}$, ultrasonicated for $1 \mathrm{~min}$ and centrifuged for $20 \mathrm{~min}$ at $13,000 \mathrm{rpm}$. The supernatants were divided in two solutions $(300 \mu \mathrm{L})$ for NMR and in vitro antiplasmodial assay.

\subsection{NMR experiments}

${ }^{1} \mathrm{H}$ NMR spectra of the plant samples were acquired on a Bruker $600 \mathrm{MHz}$ Advance II spectrometer (Bruker,
Germany) equipped with a $5 \mathrm{~mm}$ triple resonance inverse cryoprobe and a z-gradient system. Prior to data acquisition, automatic tuning and matching of the probe was performed, as well as manual shimming and automatic proton pulse calibration (pulsecal, Bruker).

${ }^{1} \mathrm{H}$ NMR analysis was performed in $3 \mathrm{~mm}$ tubes and acquired by water $1 \mathrm{D}$-water presaturation pulse sequence with composite pulses (zg30pr, Bruker) at the following parameters time domain (TD) $32 \mathrm{k}$; number of scans (NS) 64; spectral width (SW) 20 ppm; water signal irradiation point (o1) $4.84 \mathrm{ppm}$; temperature $298 \mathrm{~K}$ and relaxation delay (d1) $1.5 \mathrm{~s}$.

For two dimensional-NMR acquisition, (1) gradientselected heteronuclear single quantum coherence (HSQC) was performed by phase-sensitive ge-2D multiplicity edited HSQC using PEP and adiabatic pulses with gradients in back-inept, (2) gradient-selected heteronuclear multiple bond correlation (HMBC) was acquired by phase-sensitive ge-2D HMBC using a two-fold low-pass J-filter; and (3) J-resolved measurement was performed using a standard pulse sequence with $25 \mathrm{~Hz}$ CW-based water signal suppression. For HMBC and HSQC acquisition, each parameter of ${ }^{1} \mathrm{H}$ (f2) and ${ }^{13} \mathrm{C}$ (f1) were as follows: frequency 600.13 and $150.92 \mathrm{MHz}$, time domain (TD) $2 \mathrm{k}$ and 512 increments, spectral width (SW) 10 and $230 \mathrm{ppm}$, number of scans (NS) 64, relaxation delay (d1) $1.00 \mathrm{~s}$ and measuring temperature 298 K. For J-resolved acquisition, operating frequency, TD and SW were $600.13 \mathrm{MHz}, 16 \mathrm{k}$, and $20 \mathrm{ppm}$ for both axis (f1 and f2), respectively. Number of scans (NS) was 8; relaxation delay (d1) $2.00 \mathrm{~s}$ and temperature $298 \mathrm{~K}$. The long-range coupling constant used for HMBC was $8.0 \mathrm{~Hz}$.

\subsection{In vitro antiplasmodial activity}

In vitro antiplasmodial activity was evaluated based on parasite viability using the lactate dehydrogenase assay (Makler et al. 1993) on chloroquine-sensitive 3D7 and chloroquineresistant W2 P. falciparum strains (Murebwayire et al. 2008). Artemisinin (Sigma-Aldrich, Overijse, Belgium) was used as a positive control in all experiments, with an initial concentration of $100 \mathrm{ng} / \mathrm{mL}$. Tests were performed as described by Bero et al. (2013) with a minor modification: extracts were tested in eight-serial two-fold dilutions (concentration range: $0.78-100 \mu \mathrm{g} / \mathrm{mL}$, two wells/concentration). A statistical analysis was performed on GraphPad Prism 7.00 to compare activities of all Keetia extracts. As distributions were not Gaussian (according to D'Agostino-Pearson and Shapiro-Wilk normality tests), twigs and leaves extracts from $K$. leucantha and $K$. venos $a$ were all compared to each other with a non-parametric ANOVA (Kruskal-Wallis and Dunn's post-test, significance level of 0.05). 


\subsection{Data processing and multivariate analysis}

The ${ }^{1} \mathrm{H}$ NMR spectra were automatically reduced to ASCII files. Spectral intensities were scaled to total intensity and reduced to integrated regions of equal width $(0.04 \mathrm{ppm})$ corresponding to the region of $\delta 0.0-10.0$ by AMIX software (Bruker). The regions of $\delta 4.7-4.9$ and $\delta 3.28-3.34$ were excluded from the analysis because of the residual signal of $\mathrm{D}_{2} \mathrm{O}$ and $\mathrm{CD}_{3} \mathrm{OD}$, respectively.

The final processed data was exported in comma-separated values (.csv) and imported to SIMCA (version 15.2) software (Umetrics, Umeå, Sweden) for multivariate data analysis. All chemometric analysis were performed on the processed ${ }^{1} \mathrm{H}$ NMR data using unit variance (UV) scaling method. The OPLS-DA analysis was modeled based on (1) Keetia species (K. leucantha and K. venosa) and<smiles>[R6]OC(=O)/C=C/c1ccc(O[R6])c([R6])c1</smiles>

\section{Dihydroxy cinnamic acid moiety}

(a)

(b)

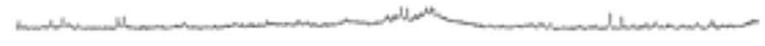

(c)

(d)

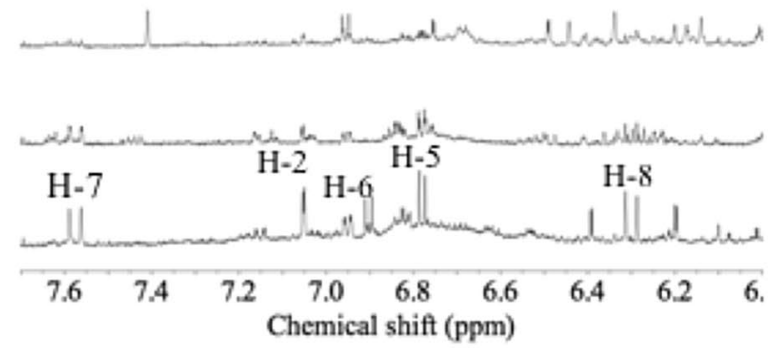

Phenolic region

Fig. 1 Chemical structures of dihydroxy cinnamic acid and triterpenoidal (oleanolic- and ursolic acids) moieties identified in Keetia species, and typical ${ }^{1} \mathrm{H} \mathrm{NMR}$ spectra $\left(600 \mathrm{MHz}, \mathrm{CH}_{3} \mathrm{OH}-d_{4}\right)$ of $\mathrm{CH}_{2} \mathrm{Cl}_{2}$ extracts of $K$. venosa twigs (a) and leaves (b), and $K$. leucantha twigs
OPLS analysis was modeled on (2) in vitro antiplasmodial activity ( $\mathrm{IC}_{50}$ values) of all Keetia samples. For metabolite elucidation, STOCSY was applied to all targeted bioactive buckets selected from the OPLS loadings (Cloarec et al. 2005). Chemical connectivity of the targeted bucket (driver peak) was analyzed by STOCSY correlation and covariance algorithm using MATLAB R2017a software (Mathworks, Natick, MA, USA). For confirmation and individual compound assessment, 2D NMR experiments (J-resolved, HMBC and HSQC) were acquired and interpreted using MestreNova 12.0.3 software17 (MestreLab Research SL, Santiago de Compostela, Spain).

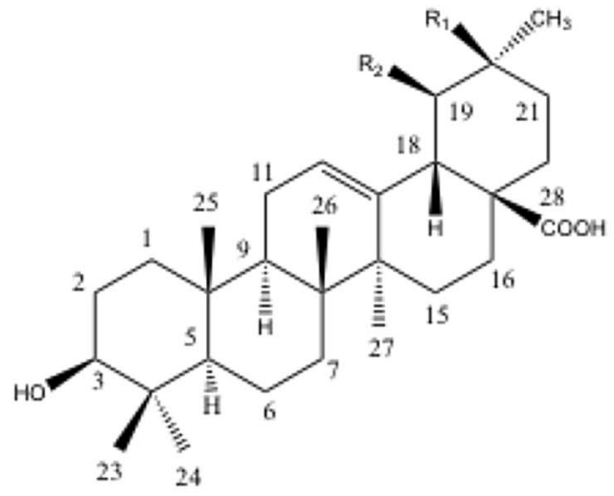

$$
\begin{aligned}
& \mathrm{R}_{1}=\mathrm{CH}_{3}, \mathrm{R}_{2}=\mathrm{H} \text {, Oleanolic acid } \\
& \mathrm{R}_{1}=\mathrm{H}, \mathrm{R}_{2}=\mathrm{CH}_{3}, \text { Ursolic acid }
\end{aligned}
$$

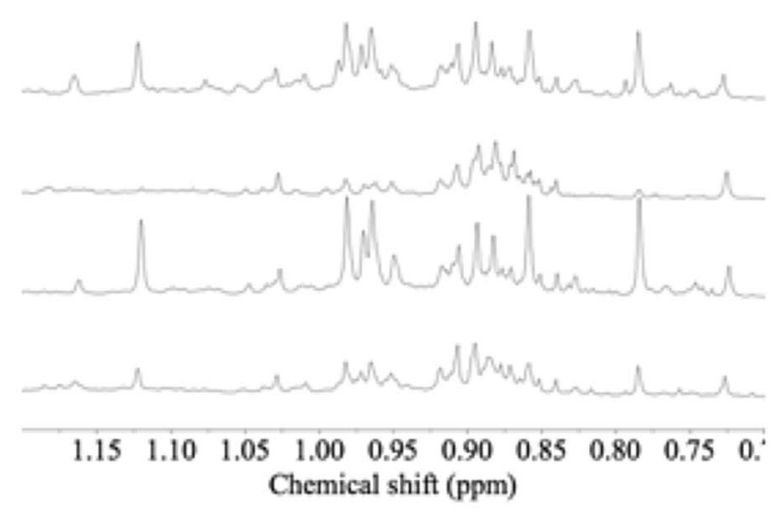

Aliphatic region

(c) and leaves (d) in phenolic ( $\delta$ 6.0-7.7) and aliphatic region $(\delta 0.7-$ 1.2). H-2, H-5, H-6, H-7 and $\mathrm{H}-8$ are $\mathrm{H}$ of dihydroxy cinnamic acid moiety 


\section{Results and discussion}

\subsection{Metabolic profiling of leaves and twigs of Keetia species by ${ }^{1} \mathrm{H}$ NMR and multivariate data analysis}

${ }^{1} \mathrm{H}$ NMR metabolic fingerprinting was applied as a highthroughput method for detecting chemical differences between Keetia samples and their related tissues. Figure 1 illustrates some representative ${ }^{1} \mathrm{H}$ NMR spectra of $\mathrm{CH}_{2} \mathrm{Cl}_{2}$ extracts of $K$. leucantha and $K$. venosa (both leaves and
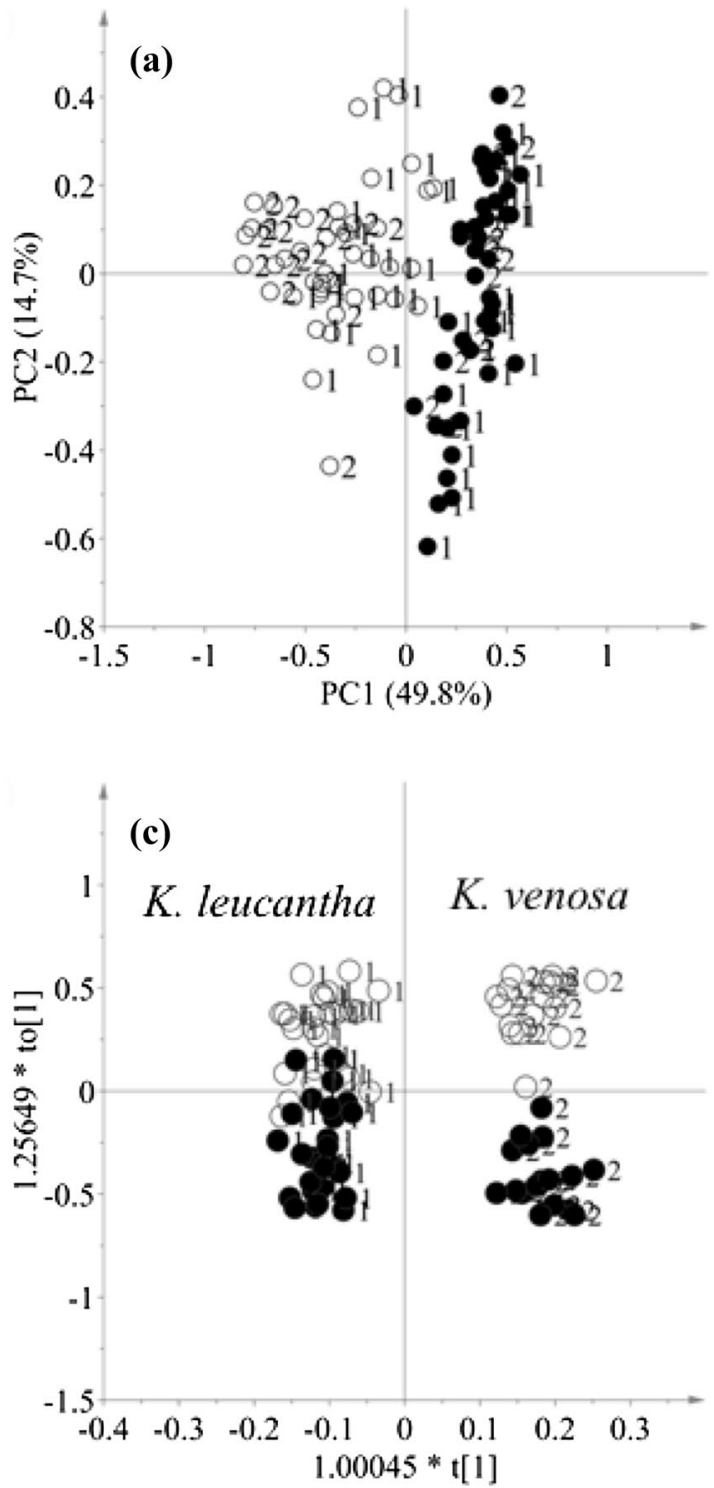

Fig. 2 Score plot $(\mathrm{PC} 1 \times \mathrm{PC} 2)(\mathbf{a})$ and $\mathrm{PC} 1$ loading plot (b) of principal component analysis of $K$. leucantha and $K$. venosa samples (leaves and twigs), and OPLDS-DA score plot (c) (t1/to1) and S-plot (d) using two species classes. 1: K. leucantha, 2: K. venosa, twigs). Two major metabolic groups were detected in the spectra, triterpenoids and phenylpropanoids. In the region of $\delta$ 0.7-1.2, many characteristic $\mathrm{CH}_{3}$ resonances were found including $\mathrm{H}-23, \mathrm{H}-24, \mathrm{H}-25, \mathrm{H}-26, \mathrm{H}-27, \mathrm{H}-29$ and $\mathrm{H}-30$ from the triterpenoids moiety. Moreover, in the region of $\delta$ 6.1-7.7, characteristic signals of dihydroxy cinnamic acid analogues, such as caffeic- or ferulic acids, were clearly detected in some Keetia samples.

Previous reports indicate that phenylpropanoids are present in both free form or conjugated to triterpenoids (Bero et al. 2013) and ${ }^{1}$ H NMR data suggest that both free- and phenylpropanoid-conjugated triterpenes are present in these
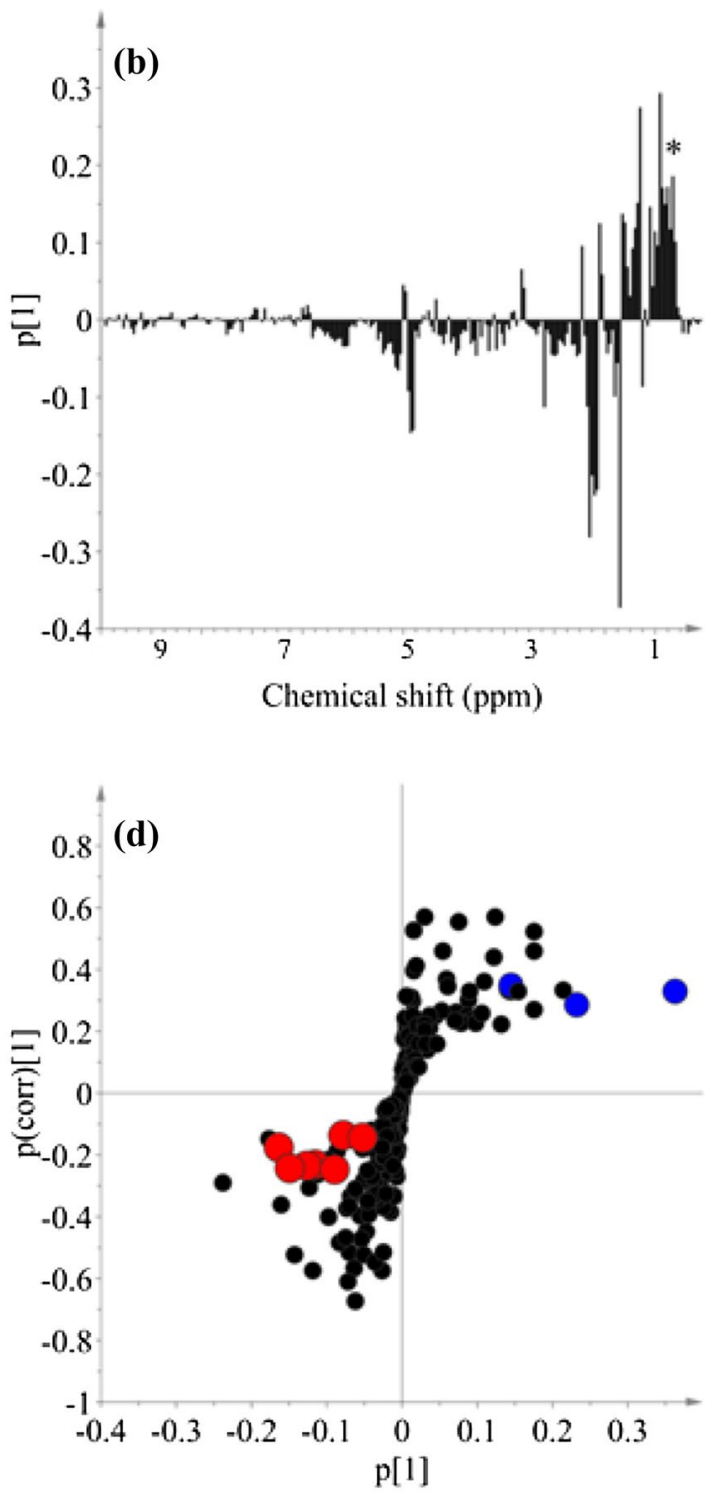

o: Leaves, $\bullet$ : twigs. *: methyl signals of triterpenoids. Red $(\bullet)$ and blue dots (•) in (d) are methyl signals of triterpenoids and squalene, respectively 
species. Phenylpropanoid conjugated triterpenes were found to be more abundant in K. leucantha twigs than in any other samples (Bero et al. 2013).

To overview the metabolites of leaves and twigs of the two Keetia species, ${ }^{1} \mathrm{H}$ NMR spectra of 88 samples were further analyzed by PCA, as shown in Fig. 2. This raw ${ }^{1} \mathrm{H}$ NMR data (bucketed) as well as their activity against two cell lines are in the Supplementary Table 1. The score plot of PCA in Fig. 2a showed that the effect of tissues on the metabolome was higher than that of species effect. The loading plot of the PCA was analyzed to identify the metabolites responsible for this discrimination between tissues. The characteristic methyl signals of triterpenoids were correlated with the positive side of PC1 loading plot in Fig. 2b, which indicates that the twigs from Keetia species were found to have higher level of triterpenoids than leaf samples.

Although the metabolic influence of species was much lower than that of tissues in PCA and not detected well using PC1 and PC2, the separation between the species might be analyzed in minor PCs. To investigate in detail the effect of species, a supervised multivariate data analysis, OPLS-DA was applied to the same data set using two classes of species (1: K. leucantha and 2: K. venosa). The score plot of OPLS-DA (Fig. 2c) showed a very clear separation and the model was well validated by permutation and CV-ANOVA tests $(\mathrm{Q} 2$ value $>0.85$ in permutation test and $\mathrm{p}$ value in CV-ANOVA $=1.4126 \mathrm{e}^{-29}$ ). The S-plot (Fig. 2d) revealed that the methyl ${ }^{1} \mathrm{H}$ resonances of triterpenoids were higher in $K$. leucantha samples than $K$. venosa. In addition, some aromatic resonances at $\delta 7.56(d, J=15.9 \mathrm{~Hz}), \delta 7.05(d$, $J=2.1 \mathrm{~Hz})$ and $\delta 6.29(d, J=15.9 \mathrm{~Hz})$ were found to be more correlated with $K$. leucantha and were elucidated as the dihydroxy cinnamic acid moieties of triterpenic esters, previously reported in this species (Bero et al. 2013). On the other hand, signals at $\delta 1.59(s), \delta 1.60(s), \delta 2.07-2.09$ $(m), \delta 5.09(t, J=7.2 \mathrm{~Hz})$ and $\delta 5.11(t, J=7.2 \mathrm{~Hz})$ were attributed to $K$. venosa samples and elucidated as an acyclic triterpene, squalene, a common secondary metabolite from the leaves of Keetia species (Bero et al. 2013; Thimmappa et al. 2014).

\subsection{In vitro antiplasmodial activity of Keetia samples and correlation with metabolomics data by OPLS}

To assess the antiplasmodial potential of the twigs and leaves, all Keetia samples were tested in vitro for their antiplasmodial activity on chloroquine-sensitive (3D7) and chloroquine-resistant (W2) strains of $P$. falciparum. The mean $\mathrm{IC}_{50}$ of leaves and twigs extracts from $K$. leucantha were $124.5 \pm 152.4 \mu \mathrm{g} / \mathrm{mL}$ and $77.9 \pm 40.6 \mu \mathrm{g} / \mathrm{mL}$ on $3 \mathrm{D} 7 \mathrm{strain}$, respectively, and $63.8 \pm 40.6 \mu \mathrm{g} / \mathrm{mL}$ and $88.7 \pm 50.7 \mu \mathrm{g} / \mathrm{mL}$ on W2 strain, respectively. In the case of $K$. venosa, the mean
$\mathrm{IC}_{50}$ for leaves and twigs extracts were $129.2 \pm 144.1 \mu \mathrm{g} /$ $\mathrm{mL}$ and $207.4 \pm 167.6 \mu \mathrm{g} / \mathrm{mL}$ on $3 \mathrm{D} 7$ strain, respectively, and $90.7 \pm 98.5 \mu \mathrm{g} / \mathrm{mL}$ and $129.9 \pm 92.7 \mu \mathrm{g} / \mathrm{mL}$ on $\mathrm{W} 2$ strain, respectively. Overall, $K$. leucantha showed similar to higher antiplasmodial activity on both strains than $K$. venosa with significant differences between both species twigs, K. leucantha leaves and $K$. venosa twigs on 3D7 $(\mathrm{p}<0.01)$ but only between leaves of $K$. leucantha and twigs of $K$. venosa on W2 $(\mathrm{p}<0.05)$. However, these activities are quite lower than artemisinin ones $\left(\mathrm{IC}_{50}=0.008 \pm 0.002\right.$ and $0.004 \pm 0.001 \mu \mathrm{g} / \mathrm{mL}$ on $3 \mathrm{D} 7$ and $\mathrm{W} 2$ strains respectively) or previously reported activities for chloroquine $\left(\mathrm{IC}_{50}=0.02 \pm 0.01\right.$ and $0.49 \pm 0.15 \mu \mathrm{g} / \mathrm{mL}$ on $3 \mathrm{D} 7$ and $\mathrm{W} 2$ strains respectively) (Bero et al. 2009). Nevertheless, crude extracts are mixtures of hundreds of compounds, some of which may have high activity, diluted with other non-active compounds. In the case of tissues among the same species, there was not much difference and the activity depended on the tested sample. Moreover, there was a high variation in the measured $\mathrm{IC}_{50}$, even in the same species and tissue. In particular, $K$. venosa samples showed a broader range of antimalarial results according to the harvest region and tissues $\left(\mathrm{IC}_{50}\right.$ from $29.18 \mu \mathrm{g} / \mathrm{mL}$ to estimated $734.86 \mu \mathrm{g} / \mathrm{mL}$ for P. falciparum 3D7 and from $30.38 \mu \mathrm{g} / \mathrm{mL}$ to estimated $439.29 \mu \mathrm{g} / \mathrm{mL}$ for P. falciparum $\mathrm{W} 2$ ).

As a next step, ${ }^{1} \mathrm{H}$ NMR data of the samples were correlated with $\mathrm{IC}_{50}$ against both strains by OPLS modeling with two $\mathrm{Y}$ variables ( $\mathrm{IC}_{50}$ on $3 \mathrm{D} 7$ and $\mathrm{W} 2$ strains). To optimize the model, two scaling methods (UV and Pareto) with Log transformation were evaluated. As shown in Table 1, the highest Q2 value was obtained from permutation test (100 permutations) when UV scaling with log transformation of $\mathrm{Y}$ variables were used. A strong correlation between metabolomics and antiplasmodial data was found in the score plot of OPLS modeling as shown in Fig. 3a, in which ${ }^{1} \mathrm{H}$ NMR data and antiplasmodial activity against both $P$. falciparum strains were used as $\mathrm{X}$ - and Y-variables with UV scaling method.

To identify metabolites responsible for the activity, a loading plot was used as shown in Fig. 3b. Some

Table 1 Q2 value of OPLS modeling of ${ }^{1} \mathrm{H}$ NMR metabolomics data and antiplasmodial activity $\left(\mathrm{IC}_{50}\right)$ against $3 \mathrm{D} 7$ and $\mathrm{W} 2 \mathrm{P}$. falciparum strains using different scaling methods and transformation obtained from permutation test with 100 permutations

\begin{tabular}{llll}
\hline Scaling method & \multicolumn{2}{l}{$\begin{array}{l}\text { Transformation of } \\
\mathrm{IC}_{50} \text { (Y-data set) }\end{array}$} & \multicolumn{2}{l}{ Q2 value } \\
\cline { 3 - 4 } & & 3D7 strain & W2 strain \\
\hline Unit variance & No & 0.277 & 0.275 \\
& Log & 0.479 & 0.473 \\
Pareto & No & 0.145 & 0.099 \\
& Log & 0.314 & 0.323 \\
\hline
\end{tabular}


Fig. 3 Score (a) and loading plot (b) of OPLS modelling with $\log \mathrm{IC}_{50}$ of in vitro antiplasmodial assay against 3D7 and W2 P. falcifarum strains t1 of ${ }^{1} \mathrm{H}$ NMR data versus $\mathrm{u} 1$ of $\log \mathrm{IC}_{50}$ of in vitro antiplasmodial activity. $\mathrm{r}^{2}$ for the correlation $=0.487$. Red bars in (b) are $\mathrm{IC}_{50}$ values against $3 \mathrm{D} 7$ and $\mathrm{W} 2$ $P$. falcifarum strains and blue bars in (b) are ${ }^{1} \mathrm{H}$ resonances of triterpenoids and phenylpropanoids associated with the activity

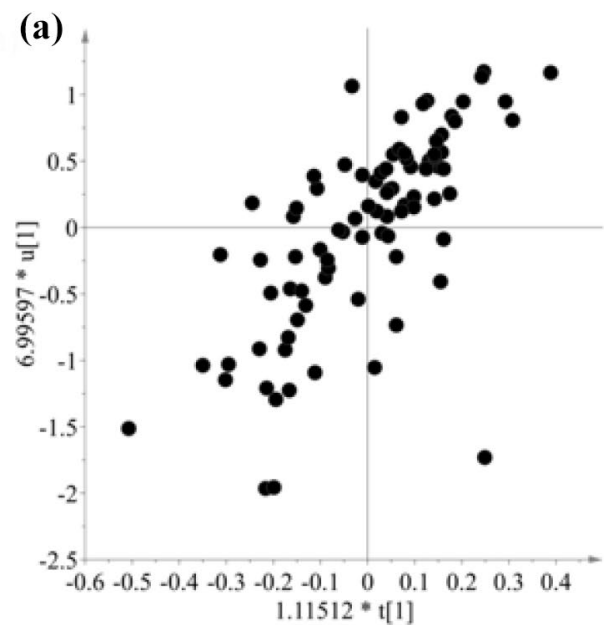

characteristic signals of triterpenoids such as methyls, H-5 around $\delta 3.2$ and $\mathrm{H}-12$ around $\delta 5.2$ were clearly related with the activity (negatively correlated with $\mathrm{IC}_{50}$ values). In addition to triterpenoidal signals, some characteristic ${ }^{1} \mathrm{H}$ resonances of phenylpropanoids were found to be associated to activity (Fig. 3b). In a previous report on the metabolites presented in K. leucantha, free forms of triterpenoids and their phenylpropanoid-conjugates were found to be major secondary metabolites, but only phenylpropanoid-conjugates displayed high antiplasmodial activity and low cytotoxicity on WI38 cells (Bero et al. 2013). Moreover, loading plot of samples with low antiplasmodial activity were also correlated to triterpenoids signals, such as methyls at around $\delta 1.60$, characteristic of the acyclic triterpene squalene (Fig. 3b).

Pentacyclic triterpenes have been already reported to show promising antimalarial potential (Beaufay et al. 2018; Isah et al. 2016) and some of them display high antiplasmodial activity and low cytotoxicity alone, in mixtures or in combination with artemisinin (Cargnin et al. 2018; Da Silva et al. 2013; Ma et al. 2008; Phillipson and O'Neill 1987). They also possess various pharmacological effects and are thought to act as multi-target compounds (Moneriz et al. 2011). Phenylpropanoid-conjugated triterpenes from $K$. leucantha have been shown to have higher antiplasmodial activity than their corresponding acids (Bero et al. 2013).

\subsection{Structure elucidation of ferulic acid conjugated triterpenoid esters}

To elucidate the detailed chemical structure of the metabolites, several 2D-NMR such as J-resolved, COSY, HSQC and HMBC (Supplementary Figs. 1, 2, 3 and 4) were firstly applied to the signals selected by OPLS modeling. The identity of each moiety of triterpene (e.g. oleanolic acid and ursolic acid) and phenylpropanoid (e.g. ferulic acid) was confirmed by 2D-NMR experiments and

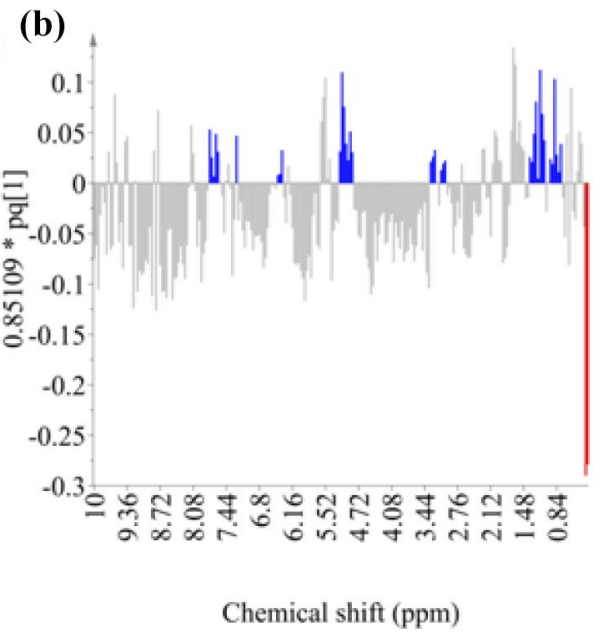

comparison with the spectra of reference compounds. However, 2D-NMR spectrum was insufficient to determine the conjugation, if any, between the two moieties, that requires the detection of a correlation band e.g. $\mathrm{H}-27$ and $\mathrm{C}=\mathrm{O}$ in $\mathrm{HMBC}$, in a very crowded region. To overcome this connectivity issue, STOCSY was used to connect the spin systems by the multicolinearity of the intensity of their signals along a set of spectra. By selecting a driver peak, also known as target peak, the STOCSY calculates the covariance and the correlation between all peaks in a dataset and creates a pseudo NMR spectrum, in which the intensities are related to the covariance values and the colors are related to the correlation values. Peaks from the same molecule or the same pathway to the driver peak are expected to have similar values, thus allowing their identification even in highly complex mixtures.

The correlation between the moieties was detected applying the STOCSY algorithm to the selected signals from the loadings (Fig. 4a). The driver peak at $\delta 7.56(d$, $J=15.9 \mathrm{~Hz}, 1 \mathrm{H}, \mathrm{CH}$ ) from the $\mathrm{H}-7$ of ferulic acid moiety strongly correlated with many terpenoidal signals, as well as other signals of ferulic acid including $\mathrm{OCH}_{3}$. The structural confirmation was based on the NMR data of the eight triterpenic esters with phenylpropanoid moieties isolated from K. leucantha twigs by Bero et al. (2013) as cited above. Two-dimensional J-resolved, HSQC and HMBC data for all the above signals indicate the presence of two phenylpropanoid conjugated triterpenes, 3 $\beta$-hydroxy-27-(E)-feruloyloxyolean-12-en-28-oic acid (1) and $3 \beta$-hydroxy-27-(E)-feruloyloxyurs-12-en-28-oic acid (2) as the major bioactive compounds present in Keetia plants (Fig. 5).

In addition, the signals of squalene in the congested region were also detected and confirmed by STOCSY (Fig. 4c). OPLS-DA revealed that these signals were strongly correlated to Keetia samples that displayed low antiplasmodial activity, in which squalene appeared 
(a)

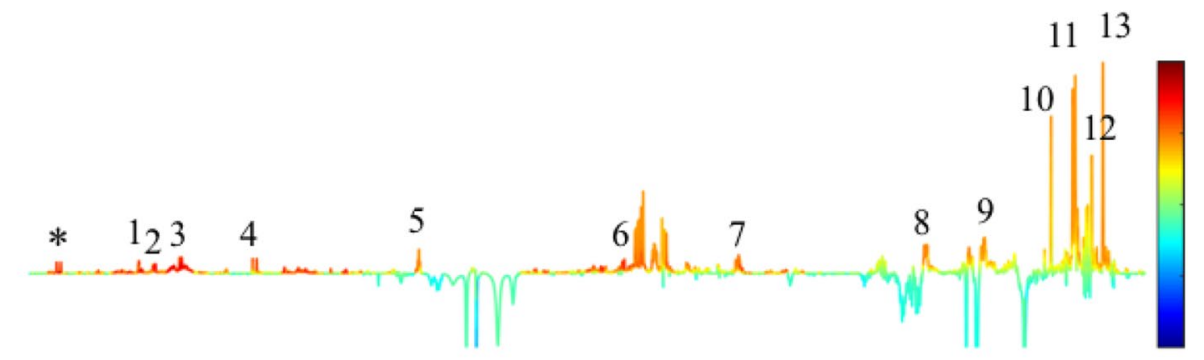

(b)

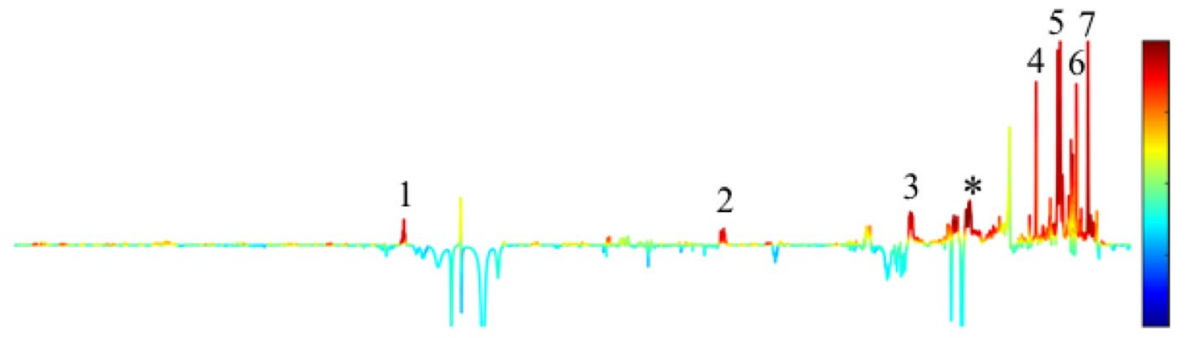

(c)

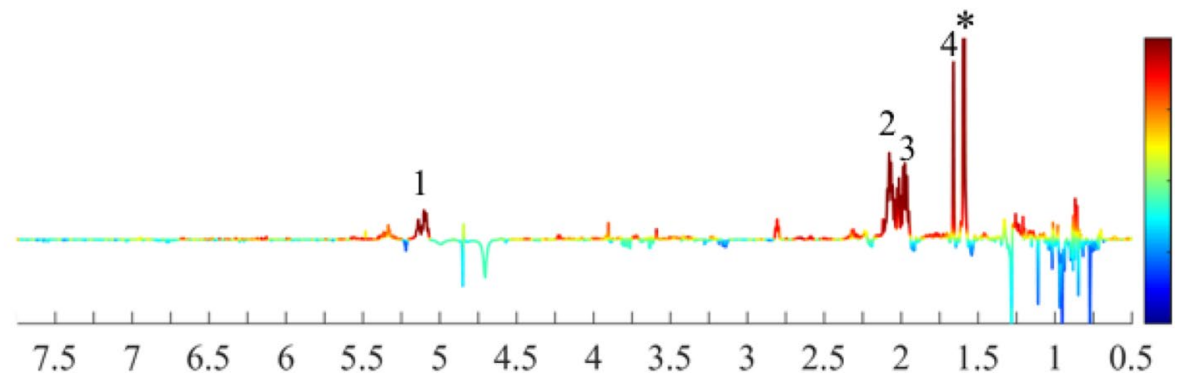

Fig. 4 STOCSY plot using drivers peak at $\delta 7.55(\mathbf{a}), \delta 1.56(\mathbf{b})$ and $\delta 1.59$ (c). Signal assignments; (a) *driver peak at $\delta 7.55(\mathrm{H}-7$ of ferulic acid moiety), 1: H-2 of ferulic acid moiety, 2: H-6 of ferulic acid moiety, 3: H-5 of ferulic acid moiety, $4: \mathrm{H}-8$ of ferulic acid moiety, 5: $\mathrm{H}-12$ of ursolic- and oleanolic acid, 6: $\mathrm{OCH}_{3}$ of ferulic acid moiety, 7: H-3 of ursolic- and oleanolic acid, 8: H-11 of ursolic- and oleanolic acid, 9: H-6 of ursolic- and oleanolic acid, 10: H-27 of ursolic- and oleanolic acid, 11: H-25 of ursolic- and oleanolic acid, 12: H-26 of ursolic- and oleanolic acid, 13: H-24 of ursolic-

as a major metabolite. The driver peak at $\delta 1.59(\mathrm{~s}, 3 \mathrm{H}$, $\mathrm{CH}_{3}$ attached to C-6) showed a high covariance and correlation with the olefinic signals at $\delta 5.11(t, J=7.2 \mathrm{~Hz}, 1 \mathrm{H}$, $\mathrm{H}-7)$ and $\delta 5.15(t, J=3.8 \mathrm{~Hz}, 1 \mathrm{H}, \mathrm{CH}, \mathrm{H}-11)$, indicating the presence of three olefinic signals with cis isomerism. Moreover, $\mathrm{CH}_{3}$ attached to C-6 also displayed high correlation with the multiplet at $\delta 1.94-2.10$ and three singlets at $\delta 1.66, \delta 1.67$, and $\delta 1.60$, connecting the olefinic signal with the complex aliphatic structure. Squalene confirmation was performed by 2D-NMR and comparison with data previously reported studies (Rotondo et al. 2017) (Fig. 5).

By comparing the STOCSY obtained through the driver peaks of compounds 1, 2 (Fig. 4a, b), it was possible to observe that both metabolites shared the same pathway, concluding that if the concentration of compound 1 increases, and oleanolic acid. (b) *Driver peak at $\delta 1.56(\mathrm{H}-6$ of ursolic- and oleanolic acid), 1: H-12 of ursolic- and oleanolic acid, 2: H-3 of ursolic- and oleanolic acid, 3: H-11 of ursolic- and oleanolic acid, 4: $\mathrm{H}-27$ of ursolic- and oleanolic acid, 5: H-25 of ursolic- and oleanolic acid, 6: H-26 of ursolic- and oleanolic acid, 7: H-24 of ursolic- and oleanolic acid. (c) *Driver peak at $\delta 1.59\left(\mathrm{CH}_{3}\right.$ attached to $\mathrm{C}-6$ of squalene), 1: H-3, H-7 and $\mathrm{H}-11$ of squalene, 2: $\mathrm{H}-4$ and $\mathrm{H}-8$ of squalene, 3: $\mathrm{H}-1$ and $\mathrm{H}-9$ of squalene, 4: $\mathrm{H}-1$ and $\mathrm{H}-2$ of squalene. For the ${ }^{1} \mathrm{H}$ assignments see the chemical structures in Figs. 1 and 5

the level of compound 2 will also increase. These results were corroborated by the loadings obtained with the multivariate data analyses based on the $\mathrm{IC}_{50}$ values that showed that both these substances contribute synergistically to the increase of the antiplasmodial activity. On the other hand, when the STOCSY plot from the squalene was compared with that of compound 1 and 2 (Fig. $4 \mathrm{a}-\mathrm{c}$ ) it was possible to observe that these compounds were displayed in opposite pathways, representing both a chemical (STOCSY) and a biological $\left(\mathrm{IC}_{50}\right)$ antagonistic effect between squalene and the cyclic triterpenes. 
Fig. 5 Chemical structures of squalene and two phenylpropanoid conjugated

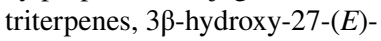
feruloyloxyolean-12-en-28-oic

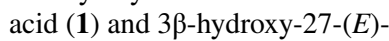
feruloyloxyurs-12-en-28-oic acid (2)<smiles>CC(C)=CCC/C(C)=C/CC/C(C)=C/CC/C=C(\C)CC/C=C(\C)CCC=C(C)C</smiles>

squalene

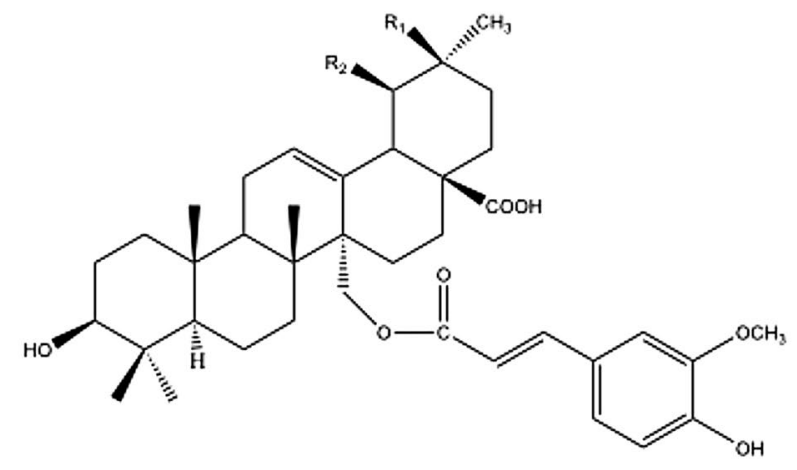

$\mathrm{R}_{1}=\mathrm{CH}_{3}, \mathrm{R}_{2}=\mathrm{H}, 3 \beta$-hydroxy-27-(E)-feruloyloxyolean-12-en-28-oic acid (1) $\mathrm{R}_{1}=\mathrm{H}, \mathrm{R}_{2}=\mathrm{CH}_{3}, 3 \beta$-hydroxy-27-(E)-feruloyloxyurs-12-en-28-oic acid (2)

\section{Conclusion}

${ }^{1} \mathrm{H}$ NMR metabolic fingerprinting was applied as a highthroughput method for the detection of chemical differences between Keetia samples (and their tissues) revealing two major metabolic groups, triterpenoids and phenylpropanoids. Unsupervised PCA analysis of the ${ }^{1} \mathrm{H}$ NMR data showed that the effect of tissues was higher than the effect of species on the metabolome and that characteristic triterpenoid methyl signals were more correlated to the twigs of both Keetia species, showing higher level of triterpenoids than the leaves. Supervised OPLS-DA based on Keetia species ( $K$. leucantha and $K$. venosa) showed a very clear separation and a validated model. The resonances of triterpenoids were found to be higher in $K$. leucantha samples than $K$. venosa. In addition, some aromatic resonances were also more correlated with $K$. leucantha and were elucidated as moieties of dihydroxy cinnamic acid derivatives of two triterpenic esters (1-2) previously reported in this species by Bero et al. (2013).

To assess the antimalarial potential of the twigs and leaves, all Keetia samples were tested for their antiplasmodial activity. In vitro biological results showed that, overall, $K$. leucantha has a similar or a little higher antimalarial activity than $K$. venosa against both chloroquine-sensitive (3D7) and chloroquine-resistant (W2) P. falciparum strains. However, in the case of tissues for a same species, there was not much difference and the activity depended on the tested sample. The ${ }^{1} \mathrm{H}$ NMR data of Keetia samples were correlated with the $\mathrm{IC}_{50}$ by OPLS modeling with two Y variables ( $\mathrm{IC}_{50}$ of 3D7 and W2 strains). To optimize the model, two scaling methods (UV and Pareto) with Log transformation were evaluated and the highest Q2 value was obtained when UV scaling with $\log$ transformation of $Y$ variables was used. A strong correlation between metabolomics data and antiplasmodial results was found in the score plot of OPLS modeling. Furthermore, characteristic signals of triterpenoids, such as methyls, $\mathrm{H}-5$ around $\delta 3.2$ and $\mathrm{H}-12$ around $\delta 5.2$ were clearly related with the activity, as well as some ${ }^{1} \mathrm{H}$ resonances of phenylpropanoids.

Overall, NMR-based metabolomics combined with supervised multivariate data analysis has proved to be a powerful strategy for the identification of bioactive metabolites in plant extracts. However, the difficulty of hyphenating NMR with chromatographic separation systems and its low-sensitivity result in spectral congestion and hamper the identification of convoluted metabolites, such as triterpenes. In this study, these problems have been solved by successfully combining STOCSY with 2D-NMR, allowing a detailed analysis of different triterpenes in the raw-extract, overcoming structure similarity and coalescence in the aliphatic region.

Although STOCY-supported NMR-based profilins could give a large improvement for the identification of metabolites, there are still limitations to fully identify a metabolite 
in mixtures due to high congested signals and similarity between analogues of a certain metabolites, e.g. terpenoids or steroids in secondary metabolites. These shortcomings could be overcome by a development of specific preparative methods for the isolation, which might be supported by mass spectrometry confirmation.

Acknowledgements The authors are grateful to Mr. Agabani (botanist of University of Abomey-Calavi, Cotonou, Benin), Dr. Gbaguidi Fernand, Dr. Gbenou Joachim, Dr. Ganfon Habib and Pr. Mansourou Moudachirou (Centre Béninois de la Recherche Scientifique et Technique, Cotonou, Benin and Laboratoire de pharmacognosie et huiles essentielles UFR pharmacie, Faculté des Sciences de la Santé, Université d'Abomey Calavi, Cotonou, Benin) for plant collections, as well as Dr Olivier Lachenaud (botanist of National Botanic Garden of Belgium, Meise, Belgium) for botanical information. We would also like to thank the Malaria's team from University of Liege for cell lines and continuous culture. The authors gratefully thank the Belgian National Fund for Scientific Research (FNRS) (Grant No. T.0190.13 and Short Stay Grant to J. Bero), Fundação de Amparo à Pesquisa do Estado de São Paulo (Grant No. 2014/05935-0 and No. 2017/06446-2), and WBI (Excellence Grant for J. Bero) for financial support.

Author contributions RTF, JB and DMS performed the metabolomic analysis. RTF interpreted the results, elucidated the metabolites and prepared the manuscript. JB, CB, AC and JQL performed the NMR and bioactivity experiments. DMS is partially involved in the identification of the metabolites and in the preparation of the manuscript. YHC designed the metabolomics research, corrected manuscript and checked data interpretation and identification. All authors reviewed and approved the manuscript.

\section{Compliance with ethical standards}

Conflict of interest The authors declare that they have no conflict of interest.

Open Access This article is distributed under the terms of the Creative Commons Attribution 4.0 International License (http://creativeco mmons.org/licenses/by/4.0/), which permits unrestricted use, distribution, and reproduction in any medium, provided you give appropriate credit to the original author(s) and the source, provide a link to the Creative Commons license, and indicate if changes were made.

\section{References}

Abreu, A. C., Coqueiro, A., Sultan, A. R., Lemmens, N., Kim, H. K., Verpoorte, R., et al. (2017). Looking to nature for a new concept in antimicrobial treatments: Isoflavonoids from Cytisus striatus as antibiotic adjuvants against MRSA. Scientific Reports, 7(1), 3777. https://doi.org/10.1038/s41598-017-03716-7.

Achan, J., Mwesigwa, J., Edwin, C. P., \& D'alessandro, U. (2018). Malaria medicines to address drug resistance and support malaria elimination efforts. Expert Review of Clinical Pharmacology. https://doi.org/10.1080/17512433.2018.1387773.

Beaufay, C., Bero, J., \& Quetin-Leclercq, J. (2018). Antimalarial terpenic compounds isolated from plants used in traditional medicine (2010-July 2016). In Natural antimicrobial agents; sustainable development and biodiversity (pp. 247-268). Cham: Springer.

Beaufay, C., Henry, G., Streel, C., Bony, E., Hérent, M. F., Bero, J., \& Quetin-Leclercq, J. (2019). Optimization and validation of antimalarial triterpenic esters in Keetia leucantha plant and plasma. Journal of Chromatography B. https://doi.org/10.1016/j. jchromb.2018.11.003.

Beaufay, C., Hérent, M. F., Quetin-Leclercq, J., \& Bero, J. (2017). In vivo anti-malarial activity and toxicity studies of triterpenic esters isolated form Keetia leucantha and crude extracts. Malaria Journal, 16(406), 1-8. https://doi.org/10.1186/s1293 6-017-2054-y.

Bero, J., Beaufay, C., Hannaert, V., Hérent, M. F., Michels, P. A., \& Quetin-Leclercq, J. (2013). Antitrypanosomal compounds from the essential oil and extracts of Keetia leucantha leaves with inhibitor activity on Trypanosoma brucei glyceraldehyde-3-phosphate dehydrogenase. Phytomedicine, 20(3-4), 270-274. https:// doi.org/10.1016/j.phymed.2012.10.010.

Bero, J., Ganfon, H., Jonville, M. C., Frédérich, M., Gbaguidi, F., DeMol, P., et al. (2009). In vitro antiplasmodial activity of plants used in Benin in traditional medicine to treat malaria. Journal of Ethnopharmacology, 122(3), 439-444. https://doi.org/10.1016/j. jep.2009.02.004.

Bero, J., Hérent, M. F., Schmeda-Hirschmann, G., Frédérich, M., \& Quetin-Leclercq, J. (2013). In vivo antimalarial activity of Keetia leucantha twigs extracts and in vitro antiplasmodial effect of their constituents. Journal of Ethnopharmacology, 149(1), 176-183. https://doi.org/10.1016/j.jep.2013.06.018.

Brennan, L. (2014). NMR-based metabolomics: From sample preparation to applications in nutrition research. Progress in Nuclear Magnetic Resonance Spectroscopy, 83, 42-49. https://doi. org/10.1016/j.pnmrs.2014.09.001.

Cardoso-Taketa, A. T., Pereda-Miranda, R., Choi, Y. H., Verpoorte, R., \& Villarreal, M. L. (2008). Metabolic profiling of the Mexican anxiolytic and sedative plant Galphimia glauca using nuclear magnetic resonance spectroscopy and multivariate data analysis. Planta Medica, 74(10), 1295-1301. https://doi. org/10.1055/s-2008-1074583.

Cargnin, S. T., Staudt, A. F., Medeiros, P., Sol, D. M. S., dos Santos, A. P. A., Zanchi, F. B., et al. (2018). Semisynthesis, cytotoxicity, antimalarial evaluation and structure-activity relationship of two series of triterpene derivatives. Bioorganic and Medicinal Chemistry Letters, 28(3), 265-272. https://doi.org/10.1016/j. bmcl.2017.12.060.

Chen, L., Yu, B., Zhang, Y., Gao, X., Zhu, L., Ma, T., \& Yang, H. (2015). Bioactivity-guided fractionation of an antidiarrheal Chinese herb Rhodiola kirilowii (Regel) maxim reveals (-)-epicatechin-3-gallate and (-)-epigallocatechin-3-gallate as inhibitors of cystic fibrosis transmembrane conductance regulator. PLoS ONE. https://doi.org/10.1371/journal.pone.0119122.

Cloarec, O., Dumas, M. E., Craig, A., Barton, R. H., Trygg, J., Hudson, J., et al. (2005). Statistical total correlation spectroscopy: An exploratory approach for latent biomarker Identification from Metabolic 1 H NMR data sets. Analytical Chemistry, 77(5), 1282-1289. https://doi.org/10.1021/ac048630x.

Da Silva, G. N. S., Maria, N. R. G., Schuck, D. C., Cruz, L. N., De Moraes, M. S., Nakabashi, M., et al. (2013). Two series of new semisynthetic triterpene derivatives: Differences in antimalarial activity, cytotoxicity and mechanism of action. Malaria Journal, 12(89), 1-7. https://doi.org/10.1186/1475-2875-12-89.

Emwas, A. H. M. (2015). The strengths and weaknesses of NMR spectroscopy and mass spectrometry with particular focus on metabolomics research. Methods in Molecular Biology, 1277, 161-193. https://doi.org/10.1007/978-1-4939-2377-9_13.

Graham, J. G., \& Farnsworth, N. R. (2010). The NAPRALERT database as an aid for discovery of novel bioactive compounds. Comprehensive Natural Products II, 3, 81-94. https://doi. org/10.1016/B978-008045382-8.00060-5.

Huang, Y., Zhang, Z., Chen, H., Feng, J., Cai, S., \& Chen, Z. (2015). A high-resolution 2D J-resolved NMR detection technique for 
metabolite analyses of biological samples. Scientific Reports, 5 , 1-9. https://doi.org/10.1038/srep08390.

Isah, M. B., Ibrahim, M. A., Mohammed, A., Aliyu, A. B., Masola, B., \& Coetzer, T. H. T. (2016). A systematic review of pentacyclic triterpenes and their derivatives as chemotherapeutic agents against tropical parasitic diseases. Parasitology, 143(10), 1219-1231. https://doi.org/10.1017/S0031182016000718.

Kim, H. K., Choi, Y. H., \& Verpoorte, R. (2010a). NMR-based metabolomic analysis of plants. Nature Protocols, 5(3), 536549. https://doi.org/10.1038/nprot.2009.237.

Kim, H. K., Saifullah, K. S., Wilson, E. G., Kricun, S. D. P., Meissner, A., et al. (2010b). Metabolic classification of South American Ilex species by NMR-based metabolomics. Phytochemistry, 71(7), 773-784. https://doi.org/10.1016/j.phyto chem.2010.02.001.

Kumar, B., Prakash, A., Ruhela, R. K., \& Medhi, B. (2014). Potential of metabolomics in preclinical and clinical drug development. Pharmacological Reports, 66(6), 956-963. https://doi. org/10.1016/j.pharep.2014.06.010.

Lei, Z., Huhman, D. V., \& Sumner, L. W. (2011). Mass spectrometry strategies in metabolomics. The Journal of Biologial Chemistry, 286(29), 25435-25442. https://doi.org/10.1074/jbc.R111.238691.

Lindon, J. C., Holmes, E., \& Nicholson, J. K. (2007). Metabonomics in pharmaceutical R \& D. The FEBS Journal, 274, 1140-1151. https://doi.org/10.1111/j.1742-4658.2007.05673.x.

Ludwig, C., \& Viant, M. R. (2010). Two-dimensional J-resolved NMR spectroscopy: Review of a key methodology in the metabolomics toolbox. Phytochemical Analysis, 21(1), 22-32. https://doi. org/10.1002/pca.1186.

Ma, C., Fred, S., Teng, G., \& Sydara, K. (2008). Study of antimalarial activity of chemical constituents from Diospyros quaesita. Chemistry and Biodiversity, 5(11), 2442-2448. https://doi.org/10.1002/ cbdv.200890209.

Makler, M. T., Ries, J. M., Williams, J. A., Bancroft, J. E., Piper, R. C., Gibbins, B. L., \& Hinrichs, D. J. (1993). Parasite lactate dehydrogenase as an assay for Plasmodium falciparum drug sensitivity. The American Society of Tropical Medicine and Hygiene, 48(6), 739-741. https://doi.org/10.4269/ajtmh.1993.48.739.

Markley, J. L., Brüschweiler, R., Edison, A. S., Eghbalnia, H. R., Powers, R., Raftery, D., \& Wishart, D. S. (2017). The future of NMR-based metabolomics. Current Opinion in Biotechnology, 43, 34-40. https://doi.org/10.1016/j.copbio.2016.08.001.

Mojab, F. (2012). Antimalarial natural products: A review. Avicenna Journal of Phytomedicine, 2(2), 52-62. https://doi.org/10.3390/ molecules15031705.

Moneriz, C., Mestres, J., Bautista, J. M., Diez, A., \& Puyet, A. (2011). Multi-targeted activity of maslinic acid as an antimalarial natural compound. FEBS Journal, 278(16), 2951-2961. https://doi.org/ 10.1111/j.1742-4658.2011.08220.x.

Murebwayire, S., Frédérich, M., Hannaert, V., Jonville, M. C., \& Duez, P. (2008). Antiplasmodial and antitrypanosomal activity of Triclisia sacleuxii (Pierre) diels. Phytomedicine, 15(9), 728-733. https ://doi.org/10.1016/j.phymed.2007.10.005.

Nagana Gowda, G. A., \& Raftery, D. (2015). Can NMR solve some significant challenges in metabolomics? Journal of Magnetic Resonance, 260, 144-160. https://doi.org/10.1016/j.jmr.2015.07.014.

Newman, D., \& Cragg, G. (2016). Natural products as sources of New drugs from 1981 to 2014. Journal of Natural Products, 79, 629661. https://doi.org/10.1021/acs.jnatprod.5b01055.

Olotu, A., Fegan, G., Wambua, J., Nyangweso, G., Leach, A., Lievens, M., et al. (2016). Seven-year efficacy of RTS,S/AS01 malaria vaccine among young African Children. New England Journal of Medicine, 374(26), 2519-2529. https://doi.org/10.1056/NEJMo a1515257.

Öman, T., Tessem, M. B., Bathen, T. F., Bertilsson, H., Angelsen, A., Hedenström, M., \& Andreassen, T. (2014). Identification of metabolites from 2D H-C HSQC NMR using peak correlation plots. BMC Bioinformatics, 15(1), 1-8. https://doi.org/10.1186/ s12859-014-0413-z.

Pezzuto, J. M. (1997). Plant-derived anticancer agents. Biochemical Pharmacology, 53(2), 121-133. https://doi.org/10.1016/S0006 -2952(96)00654-5.

Phillipson, J. D., \& O’Neill, M. J. (1987). Antimalarial and amoebicidal natural products. In K. Hostettmann \& P. J. Lea (Eds.), Biologically active natural products (pp. 49-64). Oxford: Clarendon Press.

Queiroz, E. F., Wolfender, J.-L., \& Hostettmann, K. (2009). Modern approaches in the search for new lead antiparasitic compounds from higher plants. Current Drug Targets, 10(3), 202-211. https ://doi.org/10.2174/138945009787581113.

Rotondo, A., Salvo, A., Gallo, V., Rastrelli, L., \& Dugo, G. (2017). Quick unreferenced NMR quantification of squalene in vegetable oils. European Journal of Lipid Science and Technology, 119(11), 1-6. https://doi.org/10.1002/ejlt.201700151.

Stermitz, F. R., Lorenz, P., Tawara, J. N., Zenewicz, L. A., \& Lewis, K. (2000). Synergy in a medicinal plant: Antimicrobial action of berberine potentiated by 5 '-methoxyhydnocarpin, a multidrug pump inhibitor. Proceedings of the National Academy of Sciences, 97(4), 1433-1437. https://doi.org/10.1073/pnas.030540597.

Thimmappa, R., Geisler, K., Louveau, T., O’Maille, P., \& Osbourn, A. (2014). Triterpene biosynthesis in plants. Annual Review of Plant Biology, 65(1), 225-257. https://doi.org/10.1146/annurev-arpla nt-050312-120229.

Wells, T. N. C. (2011). Natural products as starting points for future anti-malarial therapies: Going back to our roots? Malaria journal, 10(Suppl 1), S3. https://doi.org/10.1186/1475-2875-10-S1-S3.

Woodrow, C. J., \& White, N. J. (2017). The clinical impact of artemisinin resistance in Southeast Asia and the potential for future spread. FEMS Microbiology Reviews, 41(1), 34-48. https://doi. org/10.1093/femsre/fuw037.

World Health Organisation (WHO). (2018). World malaria report 2018.

Xi, Y., Ropp, J. S., Viant, M. R., Woodruff, D. L., \& Yu, P. (2008). Improved identification of metabolites in complex mixtures using HSQC NMR spectroscopy. Analytica Chimica Acta, 614(2), 127133. https://doi.org/10.1016/j.micinf.2011.07.011.Innate.

Yang, S. Y., Kim, H. K., Lefeber, A. W. M., Erkelens, C., Angelova, N., Choi, Y. H., \& Verpoorte, R. (2006). Application of two-dimensional nuclear magnetic resonance spectroscopy to quality control of ginseng commercial products. Planta Medica, 72(4), 364-369. https://doi.org/10.1055/s-2005-916240.

Yuliana, N. D., Khatib, A., Choi, Y. H., \& Verpoorte, R. (2011). Metabolomics for bioactivity assessment of natural products. Phytotherapy Research, 25(2), 157-169. https://doi.org/10.1002/ptr.3258.

Publisher's Note Springer Nature remains neutral with regard to jurisdictional claims in published maps and institutional affiliations. 